\title{
The Growth Rate of Tidally Excited Waves in Accretion Disks
}

\author{
Ethan T. Vishniac and Changsong Zhang \\ Department of Astronomy, University of Texas, Austin TX 78712 \\ I:ethan@astro.as.utexas.edu
}

\begin{abstract}
Accretion disks in close binary systems are subject to a tidally driven parametric instability which leads to the growth of internal waves near the outer edges of such disks (Goodman 1993). These waves are important in understanding the torque exerted on a disk by tidal forces and may play a role in the structure of the disk at small radii. Here we calculate the growth rate of this instability, including the effects of vertical structure and fluid compressibility. We find growth rates which are only slightly different from Goodman's original results, except that near the vertical resonance radius the growth rate can have an extremely broad and strong peak when the disk is stably stratified in the vertical direction. Higher order modes, in the sense of increasing number of vertical nodes, have similar growth rates. Our results differ from a previous calculation along these lines by Lubow et al. (1993). The difference is mostly due to their neglect of radial gradients in the tidally distorted streamlines.
\end{abstract}

\section{Introduction}

In a recent paper, Goodman (1993) pointed out that a tidally perturbed accretion disk would be subject to a parametric instability driven by the ellipticity of the distorted flow lines. This instability drives the creation of a standing wave pattern of low frequency waves with an amplitude that rises sharply near the outer edge of the disk. These waves are G-modes, or buoyancy waves, whose restoring force comes partly from vertical stratification of the fluid due to the vertical entropy gradient and partly from the radial stratification in the disk due to the steep radial gradient in specific angular momentum. Waves dominated by the former force are usually referred to as G-modes or (in a terrestrial context) internal waves. Waves dominated by the latter force are usually referred to as inertial waves. Here we will refer to them as internal waves. An overview of their properties in disks can be found in Vishniac and Diamond (1994, see also the references contained therein). 
The generation of these waves is important for at least three reasons. First, the waves created in this manner carry negative angular momentum, relative to the angular momentum of the disk, and so their generation corresponds to a torque on the disk. This torque acts as a sink for the angular momentum transported outward in an accretion disk and will be important in determining its outer radius. Second, this process will lead to a strong inward flux of such waves in regions where the tidal forces are weak. The dissipation of these waves at small radii provides a weak, but potentially significant mechanism for angular momentum transport (Vishniac \& Diamond 1989). Third, these waves can generate dynamo activity and angular momentum transport which, in an ionized disk, can dominate the angular momentum transport mediated directly by the waves (Vishniac, Jin, \& Diamond 1990, Vishniac \& Diamond 1992). This last process will be insignificant if the Balbus-Hawley instability drives a dynamo with a growth rate comparable to $\Omega$ (Balbus $\&$ Hawley 1991) but this claim is inconsistent with phenomenological models of ionized accretion disks in binary systems (see, for example Smak 1984a, Smak 1984b, Meyer \& Meyer-Hoffmeister 1984, Huang \& Wheeler 1989, Mineshige, \& Wood 1989, Mineshige \& Wheeler 1989, Cannizzo 1994, Cannizzo, Chen, \& Livio 1995).

The physical basis of instability is closely related to a three dimensional instability of an incompressible fluid with elliptical flow lines (Pierrehumbert 1986, Bayly 1986, Landman, \& Saffman 1987). An observer moving with the fluid in an accretion disk will see the tidal forces as inducing a set of time-dependent stresses whose comoving frequency, $\bar{\omega}$, is $m\left(\Omega-\Omega_{b}\right)$, where $m$ is the dimensionless azimuthal wavenumber of the tidal force, $\Omega$ is the local orbital frequency in the disk, and $\Omega_{b}$ is the binary orbital frequency. A realistic tidal force will have components at all values of $m$, but the strongest components will have small values of $m$. If we consider the $m=2$ component then we see that an internal wave with $\bar{\omega}=\Omega-\Omega_{b}$ and $m=1$ can interact with this component of the tidally induced stress to produce a wave traveling in the opposite direction, with the same $m$ and $\bar{\omega}$. In Goodman's paper he showed that this process could lead to exponential growth as each component of the standing wave amplifies the other. Since this process is inherently local no special boundary conditions are required. It is necessary that the waves be confined to the midplane of the disk, since the waves will need to maintain coherence over a radial distance comparable to their group velocity divided by the instability growth rate, but internal waves are naturally confined in this manner.

The significance of this instability depends on its growth rate. A small growth rate not only implies weaker waves, but makes the instability vulnerable to suppression from dissipative effects, including turbulent dissipation from any other instabilities present. Goodman (1993) calculated the growth rate to leading order in the tidal forces for an adiabatic fluid in a disk without vertical structure assuming that the induced fluid motions 
were incompressible. He found that at small $r$ the growth rate was given by

$$
\sigma(r) \approx \frac{15}{4} \frac{G M_{2}}{a^{3} \Omega(r)},
$$

where $M_{2}$ is the mass of the secondary (mass-losing) star, and $a$ is the semi-major axis of the binary orbit. This result is consistent with the growth rate found by Ryu and Goodman (1994), who used a different approach and who also carried out a two dimensional simulation to look at the nonlinear saturation of the instability. Goodman also found a singularity in the growth rate near the vertical resonance discovered by Lubow (1981). This resonance occurs at the radius where

$$
\Omega(r)=\frac{\Omega_{b}}{1-(\gamma+1)^{1 / 2} / 2},
$$

where $\gamma$ is the adiabatic index of the gas in the disk. This singularity is due to pumping of the waves by vertical motions rather than the ellipticity of the flow lines, but is a natural part of the parametric instability in accretion disks.

More recently, Lubow, Pringle, and Kerswell (1993) repeated the growth rate calculation including the effects of an isothermal vertical structure. They found a reduced growth rate at all radii, by about an order of magnitude, which they ascribed to the stabilizing effects of the vertical stratification. However, their calculation differed in other respects as well. In particular, they neglected vertical motions, which eliminated the singularity, and they treated the flow lines as locally self-similar, which eliminated radial derivatives of the tidal distortions. They also suggested that the instability could be suppressed by a suitable choice of radial boundary conditions, but further work by Ryu et al. (1995) has shown that the instability is purely local and relatively insensitive to radial boundaries.

In this paper we present an improved calculation of the linear growth rate of this instability, including the effects of vertical structure, compressibility, and variations in the local adiabatic index $\gamma$, while retaining tidally induced vertical motions and radial derivatives of flow lines. We show that a full calculation is reasonably consistent with Goodman's original estimate for the growth rate of the parametric instability in a realistic disk. However, there is one interesting difference. Vertical stratification greatly increases the amplitude and width of the peak in the growth rate associated with the vertical resonance.

In $\S 2$ we derive the necessary formulae for calculating the linear growth rate. In $\S 3$ we present our results for disks with different vertical structures and adiabatic indices and compare our results with previous work. In $\S 4$ we discuss the implications of this work and some directions for future research. 


\section{The Growth Rate Formalism}

The response of an accretion disk to tidal perturbations is an example of the more general situation where one has a set of modes of a system and one perturbs the evolution operator. In this case the basic modes are the $m= \pm 1$ standing internal waves of an accretion disk and the perturbation is the change in the evolution operator for these waves due to the tidal distortions. If a frequency eigenstate of the system is represented by some state vector $A_{i}$ then its unperturbed evolution is given by

$$
\partial_{t} A_{i}=L_{i j} A_{j}=i \bar{\omega} A_{j}
$$

where we have used implicit summation and $\bar{\omega}$ is the comoving frequency, i.e. we treat the mode evolution from the point of view of an observer moving with the fluid. It is useful to define an adjoint state vector and corresponding eigenmodes, $\tilde{A}_{i}$, using the adjoint of $L_{i j}$, i.e.

$$
\partial_{t} \tilde{A}_{i}=-L_{i j}^{\dagger} \tilde{A}_{j}=i \bar{\omega} \tilde{A}_{j}
$$

When $i L$ is a hermitian operator the two sets of eigenvectors are identical. We can define an inner product, $\langle\tilde{A} A\rangle$, in the usual way as

$$
\langle\tilde{A} A\rangle \equiv \int \tilde{A}_{i}^{*} A_{i} d V
$$

where the integral is over the volume of the system.

When the system is perturbed so that $L \rightarrow L+\Delta L$ then the eigenfunctions are perturbed as well. We can invoke the normalization constraint, i.e.

$$
\langle\tilde{A} A\rangle=\langle(\tilde{A}+\tilde{\Delta})(A+\Delta)\rangle
$$

to show that

$$
\langle\tilde{A}+\tilde{\Delta}|L+\Delta L| A+\Delta\rangle=i(\bar{\omega}+\delta \omega)\langle\tilde{A} A\rangle=i \bar{\omega}\langle\tilde{A} A\rangle+\langle\tilde{A}|\Delta L| A\rangle
$$

or

$$
i \delta \omega=\frac{\langle\tilde{A}|\Delta L| A\rangle}{\langle\tilde{A} A\rangle} .
$$

In order to solve for the growth rate we need to find the unperturbed eigenvectors $A$ and $\tilde{A}$ and calculate the matrix elements due to tidal perturbations of the disk.

We will assume that we are dealing with standing waves whose radial structure can be approximated by a plane wave of the form $\cos \left(k_{r} r+\phi\right)$, where $\phi$ is an arbitrary phase 
function, and whose azimuthal wavenumber is $\pm 1 / r$. The latter is a requirement for an exact resonance with the $m=2$ component of the tidal force. More generally we do not need an exact resonance to drive the instability, but we will restrict ourselves to that case in this paper. Waves with a low $k_{\theta} r \equiv m$ of order unity will show secular radial variations on scales $\sim r / m$ (Vishniac \& Diamond 1989). As long as we restrict ourselves to $k_{r} \gg k_{\theta}$, as we do here, then we can ignore this effect.

We will restrict ourselves to adiabatic perturbations of a disk with arbitrary vertical structure. The evolution of radial plane wave perturbations of the disk, with radial wavenumber $k_{r}$, are described by the following equation.

$$
\frac{d}{d t}\left(\begin{array}{c}
v_{r} \\
v_{\theta} \\
v_{z} \\
\chi \\
\delta
\end{array}\right)=\left(\begin{array}{ccccc}
0 & 2 \Omega & 0 & -S i k_{r} & 0 \\
-\Omega / 2 & 0 & 0 & 0 & 0 \\
0 & 0 & 0 & -S\left[(\ln (S \rho))^{\prime}+\partial_{z}\right] & -z \Omega^{2} \\
-\frac{c_{s}^{2}}{S} i k_{r} & 0 & \frac{1}{S}\left(z \Omega^{2}-c_{s}^{2} \partial_{z}\right) & 0 & 0 \\
-i k_{r} & 0 & -\left(\ln (\rho)^{\prime}+\partial_{z}\right) & 0 & 0
\end{array}\right)\left(\begin{array}{c}
v_{r} \\
v_{\theta} \\
v_{z} \\
\chi \\
\delta
\end{array}\right)
$$

where a prime denotes a derivative in the $\hat{z}$ direction, $S \equiv P^{1 / \gamma} / \rho, c_{s}(z)$ is the sound speed, $\rho(z)$ is the density, $P(z)$ is the pressure, $\delta$ is the fractional density perturbation, and $\chi$ is the pressure perturbation divided by $P^{1 / \gamma}$.

If we take the perturbation to have a well-defined comoving frequency, consistent with our approximation of the perturbation as a plane wave, we get the following second order equation in $z$ for $\chi$

$$
\left(\chi^{\prime} \ln \left[\frac{S^{2} \rho}{N^{2}-\bar{\omega}^{2}}\right]\right)^{\prime}+\left(\bar{\omega}^{2}-N^{2}\right)\left(\frac{1}{c_{s}^{2}}+\frac{k_{r}^{2}}{\Omega^{2}-\bar{\omega}^{2}}\right) \chi=0,
$$

where $N^{2}$ is the square of the Brunt-Väisälä frequency, defined by

$$
N^{2} \equiv z \Omega(\ln S)^{\prime}
$$

This equation has an apparent singularity when $\bar{\omega}^{2}=N^{2}$, but closer examination shows that there are always two linearly independent and regular solutions passing through this point. The only boundary condition is the requirement that $\chi$ vanish as $|z| \rightarrow \infty$. The other variables can be found in terms of $\chi$ using equation (9). Since terms of order $m /\left(r k_{r}\right)$ are negligible, they are

$$
\begin{gathered}
v_{r}=\frac{-k_{r} \bar{\omega} S}{\bar{\omega}^{2}-\Omega^{2}} \chi, \\
v_{\theta}=\frac{-i k_{r} S}{\bar{\omega}^{2}-\Omega^{2}} \frac{\Omega}{2} \chi,
\end{gathered}
$$




$$
v_{z}=\frac{i \bar{\omega} S}{\bar{\omega}^{2}-N^{2}} \partial_{z} \chi
$$

and

$$
\delta=\frac{\partial_{z} S}{\bar{\omega}^{2}-N^{2}} \partial_{z} \chi+\frac{S}{c_{s}^{2}} \chi
$$

There will be an infinite number of solutions that satisfy this boundary condition, just as there are an infinite number of harmonics of the resonant mode of a box. However, modes with many vertical nodes, or alternatively modes with large $k_{z}$, will be more easily saturated due to nonlinear processes. Here we will consider only a few of the lowest order modes. In order to study the parametric instability we need to look at the standing wave pattern formed by the superposition of outgoing and ingoing waves. For a given $\bar{\omega}$ and $k_{r}$ this involves an overall phase factor of the form

$$
\chi \propto \cos \left(k_{r} r+\phi\right) \cos (\bar{\omega} t+\theta+\psi)
$$

where $\phi$ and $\psi$ are phase constants and $\theta$ is the azimuthal coordinate. The angle $\phi$ is irrelevant and may vary from one annular region to another. (The instability only requires a limited amount of radial coherence.) Phase factors for the other components of the state vector can be derived from equations (12) through (15). Given a model for the vertical structure of a disk the vertical eigenfunctions can be calculated from these equations in a straightforward manner.

The evolution of the adjoint state vector is given by

$$
\frac{d}{d t}\left(\begin{array}{c}
\tilde{v}_{r} \\
\tilde{v}_{\theta} \\
\tilde{v}_{z} \\
\tilde{\chi} \\
\tilde{\delta}
\end{array}\right)=\left(\begin{array}{ccccc}
0 & \frac{\Omega}{2} & 0 & -i k_{r} \frac{c_{s}^{2}}{S} & -i k_{r} \\
-2 \Omega & 0 & 0 & 0 & 0 \\
0 & 0 & 0 & -\frac{(2-\gamma)}{S} z \Omega^{2}-\frac{c_{s}^{2}}{S} \partial_{z} & (\ln \rho)^{\prime}-\partial_{z} \\
-i k_{r} S & 0 & S\left((\ln \rho)^{\prime}-\partial_{z}\right) & 0 & 0 \\
0 & 0 & z \Omega^{2} & 0 & 0
\end{array}\right)\left(\begin{array}{c}
\tilde{v}_{r} \\
\tilde{v}_{\theta} \\
\tilde{v}_{z} \\
\tilde{\chi} \\
\tilde{\delta}
\end{array}\right),
$$

where the tilde variables are defined by this equation. Note that we have used the relation

$$
\partial_{z}\left(c_{s}^{2} / S\right)=-\frac{(\gamma-1)}{S} z \Omega^{2}
$$

in deriving equation (17). The vertical structure equation, analogous to equation (10) is

$\partial_{z}^{2}\left(\frac{c_{s}^{2}}{S} \tilde{\chi}\right)-\partial_{z}\left(\frac{c_{s}^{2}}{S} \tilde{\chi}\right)(\ln (G \rho))^{\prime}+\frac{c_{s}^{2}}{S} \tilde{\chi}\left(\frac{-\Omega^{2}}{c_{s}^{2}} G+\frac{k_{r}^{2} \Omega^{2}}{\bar{\omega}^{2}-\Omega^{2}}\left(1-\frac{\bar{\omega}^{2}}{\Omega^{2}}+z \partial_{z}\left(\ln \left(\frac{z}{G \rho}\right)\right)\right)\right)=0$,

where

$$
G \equiv 1-\frac{\bar{\omega}^{2}}{\Omega^{2}}-\frac{z^{2} k_{r}^{2} \Omega^{2}}{\Omega^{2}-\bar{\omega}^{2}}
$$


Equation (19) has an apparent singularity at $G=0$. However, as before, one can show that there exists two linearly independent and well-behaved solutions that pass through this point. The other adjoint variables are given by

$$
\begin{gathered}
\tilde{v}_{r}=\frac{-k_{r} \bar{\omega}}{\bar{\omega}^{2}-\Omega^{2}}\left(\frac{c_{s}^{2} \tilde{\chi}}{S}\left(1-\frac{\Omega^{2} k_{r}^{2} z^{2}}{G\left(\bar{\omega}^{2}-\Omega^{2}\right)}\right)-\frac{z}{G} \partial_{z}\left(\frac{c_{s}^{2}}{S} \tilde{\chi}\right)\right), \\
\tilde{v}_{\theta}=\frac{-i 2 k_{r} \Omega}{G\left(\bar{\omega}^{2}-\Omega^{2}\right)}\left(\frac{c_{s}^{2}}{S} \tilde{\chi}\left(1-\frac{\bar{\omega}^{2}}{\Omega^{2}}\right)-z \partial_{z}\left(\frac{c_{s}^{2}}{S} \tilde{\chi}\right)\right), \\
\tilde{v}_{z}=\frac{-i \bar{\omega}}{\Omega^{2} G}\left(\left(\frac{c_{s}^{2} \tilde{\chi}}{S}\right) \frac{\Omega^{2} k_{r}^{2} z}{\bar{\omega}^{2}-\Omega^{2}}+\partial_{z}\left(\frac{c_{s}^{2}}{S} \tilde{\chi}\right)\right),
\end{gathered}
$$

and

$$
\tilde{\delta}=-\frac{z}{G}\left(\left(\frac{c_{s}^{2} \tilde{\chi}}{S}\right) \frac{\Omega^{2} k_{r}^{2} z}{\bar{\omega}^{2}-\Omega^{2}}+\partial_{z}\left(\frac{c_{s}^{2}}{S} \tilde{\chi}\right)\right) .
$$

These equations can be solved, once again invoking $\tilde{\chi} \rightarrow 0$ as $|z| \rightarrow \infty$. Alternatively, we can notice that there is a close relationship between the state vector and the adjoint state vector. The fact that $\langle\tilde{A} A\rangle$ is a constant suggests that we should be able to equate the state norm with some conserved state property, e.g. the energy. In fact, if we compare $\tilde{v}_{r}$ and $(1 / 2) \rho v_{r}$ then we can show that equations (10), (12), (19), and (21) imply that the two variables satisfy the same second order differential equation in $z$. The boundary condition at $|z| \rightarrow \infty$ does not distinguish between solutions that differ only by a complex factor, but the phase and amplitude of the adjoint state vector is undefined in any case. We conclude that we can take $\tilde{v}_{r}=(1 / 2) \rho v_{r}$. With this choice, we can use equations (12) through (15) and (21) through (24) to show that

$$
\left(\begin{array}{c}
\tilde{v}_{r} \\
\tilde{v}_{\theta} \\
\tilde{v}_{z} \\
\tilde{\chi} \\
\tilde{\delta}
\end{array}\right)=\left(\begin{array}{c}
\frac{1}{2} \rho v_{r} \\
2 \rho v_{\theta} \\
\frac{1}{2} \rho v_{z} \\
\frac{1}{2} \rho\left(\frac{S^{2}}{c_{s}^{2}} \chi-\frac{S}{c_{s}^{2}} z \Omega^{2} \Delta z\right) \\
\frac{1}{2} \rho z \Omega^{2} \Delta z
\end{array}\right),
$$

where

$$
\Delta z \equiv \frac{v_{z}}{i \bar{\omega}} .
$$

The meaning of this choice is clearer if we note that equation (25) implies that

$$
\langle\tilde{A} A\rangle=\frac{1}{2} \rho\left(\left|v_{r}\right|^{2}+4\left|v_{\theta}\right|^{2}+\left|v_{z}\right|^{2}\right)+\frac{|\delta P|^{2}}{2 \rho c_{s}^{2}}-\frac{1}{2} \rho z \Omega^{2} \Delta z^{*}\left(\frac{\delta P}{\gamma P}-\delta\right) .
$$


Furthermore

$$
\begin{array}{rlc}
-\frac{1}{2} \rho z \Omega^{2} \Delta z^{*}\left(\frac{\delta P}{\gamma P}-\delta\right) & = & -\frac{1}{2} \rho z \Omega^{2} \Delta z^{*}\left(\frac{\delta S}{S}\right) \\
& = & -\frac{1}{2} \rho z \Omega^{2} \Delta z^{*}\left(-\Delta z \partial_{z} \ln S\right) \\
& = & \frac{1}{2} \rho N^{2}|\Delta z|^{2}
\end{array}
$$

Therefore,

$$
\langle\tilde{A} A\rangle=\frac{1}{2} \rho\left(\left|v_{r}\right|^{2}+4\left|v_{\theta}\right|^{2}+\left|v_{z}\right|^{2}\right)+\frac{|\delta P|^{2}}{2 \rho c_{s}^{2}}+\frac{1}{2} \rho N^{2}|\Delta z|^{2},
$$

which is to say that using this convention the norm of the state is the locally measured energy density. Goodman (1993) used the local energy density in his calculation of the perturbation growth rate, but, consistent with his neglect of compressibility and vertical structure, left out the last two terms.

It remains to find the operator $\Delta L$ and calculate the matrix elements $\langle\tilde{A}|\Delta L| A\rangle$. We will start with the lagrangian displacements produced by the tidal forces. We follow Goodman in using first-order epicyclic theory to calculate the radial and angular displacements. The radial and azimuthal displacements are

$$
\xi_{r}=\frac{1}{4 \bar{\omega}^{2}-\Omega^{2}}\left(\frac{d \Phi_{2}}{d r}+\frac{2 \Omega}{r \bar{\omega}} \Phi_{2}\right) \cos (2 \bar{\omega} t+2 \theta)
$$

and

$$
\xi_{\theta}=\frac{-1}{2\left(4 \bar{\omega}^{2}-\Omega^{2}\right)}\left(\frac{2 \Omega}{r \bar{\omega}} \frac{d \Phi_{2}}{d r}+\frac{4 \bar{\omega}^{2}+3 \Omega^{2}}{r^{2} \bar{\omega}^{2}} \Phi_{2}\right) \sin (2 \bar{\omega} t+2 \theta),
$$

where $\bar{\omega}=\Omega-\Omega_{b}, \theta$ is measured relative to the axis running between the two stars, and $\Phi_{2}$ is the azimuthal $m=2$ Fourier component of the tidal potential. It is given by Brouwer \& Clemence 1961)

$$
\Phi_{2}=-q \frac{G M_{1}}{a} b_{2}^{(1 / 2)}(r / a),
$$

where $q$ is the mass ratio for the system, $M_{1}$ is the primary stellar mass, and $b_{2}^{1 / 2}(r / a)$ is a Laplace coefficient. The vertical displacement follows from examining the response of the disk to long radial wavelength perturbations to the local vertical gravity. This gives

$$
\xi_{z}=\frac{z \cos (2 \bar{\omega}+2 \theta)}{4 \bar{\omega}^{2}-(\gamma+1) \Omega^{2}}\left((\gamma-1) \Omega^{2}\left(\frac{1}{r} \partial_{r}\left(r \xi_{r}\right)+\frac{1}{r} \partial_{\theta} \xi_{\theta}\right)-\frac{1}{r} \frac{d}{d r}\left(\frac{d \Phi_{2}}{d r}\right)+\frac{4}{r^{2}} \Phi_{2}-\frac{3 \xi_{r}}{r} \Omega^{2}\right) .
$$

This differs from the expression in Goodman (1993) in two respects. First, the sign of the $\Phi_{2}$ terms are reversed. Second, there is an additional term proportional to $\xi_{r}$ which comes from the change in vertical gravity induced by radial motions. Writing these displacements 
as a set of coefficients of the angular functions $\cos (2 \bar{\omega} t+2 \theta)$ and $\sin (2 \bar{\omega} t+2 \theta)$ we find the tidally induced eulerian velocity perturbations are

$$
\begin{aligned}
\Delta v_{r} & =\left(\Omega \xi_{\theta}-2 \bar{\omega} \xi_{r}\right) \sin (2 \bar{\omega} t+2 \theta) \\
\Delta v_{\theta} & =\left(\frac{\Omega}{2} \xi_{r}+2 \bar{\omega} \xi_{\theta}\right) \cos (2 \bar{\omega} t+2 \theta)
\end{aligned}
$$

and

$$
\Delta v_{z}=-2 \bar{\omega} \xi_{z} \sin (2 \bar{\omega} t+2 \theta)
$$

In addition, we need to know the tidally induced perturbations to the pressure, density, and entropy. These are

$$
\begin{array}{rlc}
\frac{\Delta S}{S} & = & -\xi \partial_{z} \ln S \\
= & \frac{-\xi_{z} N^{2}}{z \Omega^{2}} \\
= & -\frac{\partial_{z} \xi_{z}}{\Omega^{2}} N^{2} \cos (2 \bar{\omega} t+2 \theta) \\
\frac{\Delta \rho}{\rho}= & & -\vec{\nabla} \cdot \vec{\xi}-\xi_{z} \partial_{z} \ln \rho \\
=-\left(\frac{1}{r} \partial_{r}\left(r \xi_{r}\right)+\frac{2}{r} \xi_{\theta}+\partial_{z} \xi_{z}\left(1+z \partial_{z} \ln \rho\right)\right) \cos (2 \bar{\omega} t+2 \theta)
\end{array}
$$

and

$$
\frac{\Delta P}{P}=\gamma\left(\frac{\Delta S}{S}+\frac{\Delta \rho}{\rho}\right)
$$

Here we have used the assumption that the tidal perturbations are adiabatic. This assumption may not always be valid, but since we have already employed it in deriving the dispersion relation for the waves, we have not further compromised our results by using it here. In any case, this assumption should be reasonable as long as the cooling time for the disk is much greater than the orbital time. The radial and vertical derivatives of these expressions can be calculated in a straightforward manner.

It is convenient to rewrite the components of the state vectors as coefficients of phase factors, following equation (16). For both the state vector and its adjoint the phase factors have the form

$$
\left(\begin{array}{c}
\sin \left(k_{r} r+\phi\right) \sin (\bar{\omega} t+\theta+\psi) \\
-\sin \left(k_{r} r+\phi\right) \cos (\bar{\omega} t+\theta+\psi) \\
-\cos \left(k_{r} r+\phi\right) \sin (\bar{\omega} t+\theta+\psi) \\
\cos \left(k_{r} r+\phi\right) \cos (\bar{\omega} t+\theta+\psi) \\
\cos \left(k_{r} r+\phi\right) \cos (\bar{\omega} t+\theta+\psi)
\end{array}\right) .
$$

The definitions of the state vector and adjoint state vector components are then modified by dividing through by $i$ for every factor of $\bar{\omega}$ or $k_{r}$. Note that when we are done constructing 
our standing wave patterns there is no distinction left between $\tilde{A}$ and $\tilde{A}^{*}$. Finally, we note that matrix elements which involve products of the form $\cos \left(k_{r} r\right) \sin \left(k_{r} r\right)$ will not vanish exactly. Instead,

$$
\left\langle\sin \left(k_{r} r\right) F(r) \cos \left(k_{r} r\right)\right\rangle=-\frac{1}{4}\left\langle k_{r}^{-1} \partial_{r} F(r)\right\rangle .
$$

When the matrix element involves a factor of $k_{r}$ such terms will contribute to the total growth rate at the same order as other terms. This point is particularly significant since the radial gradients of the tidal perturbations can be much larger than the tidal perturbations divided by $r$. We note that in performing the necessary integration by parts we have neglected secular radial variations in the eigenmodes, which will arise from radial variations in density and pressure.

We are now ready to write down expressions for the matrix elements which enter into equation (8). The tidally perturbed radial acceleration equation is

$$
\begin{gathered}
\partial_{t} v_{r}=2 \Omega v_{\theta}-\frac{1}{\rho} \partial_{r} P-\left(\Delta v_{r} \partial_{r}+\frac{\Delta v_{\theta}}{r} \partial_{\theta}+\Delta v_{z} \partial_{z}+\partial_{r} \Delta v_{r}\right) v_{r}+\left(\frac{2 \Delta v_{\theta}}{r}-\frac{1}{r} \partial_{\theta} \Delta v_{r}\right) v_{\theta} \\
-v_{z} \partial_{z} \Delta v_{r}+\frac{\Delta \rho}{\rho} S \partial_{r} \chi+\frac{1}{\rho} \partial_{r} \Delta P .
\end{gathered}
$$

Taking into account the radial phase factors given in equation (16) we can use this to write the top row of $\Delta L$ as

$$
\Delta L_{r i}=\left(-\frac{1}{2} \partial_{r} \Delta v_{r}-\Delta v_{z} \partial_{z}-\frac{\Delta v_{\theta}}{r} \partial_{\theta}, \frac{2 \Delta v_{\theta}}{r}-\frac{1}{r} \partial_{\theta} \Delta v_{r}, 0, \frac{\Delta \rho}{\rho} S \partial_{r}, 0\right) .
$$

This gives a contribution to the growth rate, following equation (8) of

$$
\begin{aligned}
& \left\langle\tilde{v}_{r}|\Delta L| A\right\rangle=\quad \frac{\sin (2 \psi)}{8} \int_{-\infty}^{\infty} \tilde{v}_{r}\left(v_{r}\left(-\frac{\partial_{r} \Delta v_{r}}{2}-\frac{\Delta v_{\theta}}{r}\right)-\Delta v_{z} \partial_{z} v_{r}-\left(\frac{2 \Delta v_{\theta}}{r}-\frac{2 \Delta v_{r}}{r}\right) v_{\theta}\right. \\
& \left.-\chi k_{r} \frac{\Delta \rho}{\rho} S\right) d z \\
& =\frac{\sin (2 \psi)}{8} \int_{-\infty}^{\infty} \tilde{v}_{r}\left(v_{r}\left(\bar{\omega} \partial_{r} \xi_{r}-\frac{\Omega}{2} \partial_{r} \xi_{\theta}+\left(\frac{3}{4} \Omega-2 \bar{\omega}\right) \frac{\xi_{\theta}}{r}-2 \Omega \frac{\xi_{r}}{r}\right)+\partial_{z} v_{r} 2 \bar{\omega} \xi_{z}\right. \\
& -\frac{2 v_{\theta}}{r}\left(\xi_{r}\left(\frac{\Omega}{2}+2 \bar{\omega}\right)+\xi_{\theta}(2 \bar{\omega}-\Omega)\right) \\
& \left.+\chi k_{r} S\left(\frac{\xi_{r}}{r}+\partial_{r} \xi_{r}+\frac{2}{r} \xi_{\theta}+\partial_{z} \xi_{z}\left(1+z \partial_{z} \ln \rho\right)\right)\right) d z \text {. }
\end{aligned}
$$

Similarly, the tidally perturbed azimuthal acceleration equation is

$$
\partial_{t} v_{\theta}=-\frac{\Omega}{2} v_{r}-\left(\partial_{r} \Delta v_{\theta}+\frac{\Delta v_{\theta}}{r}\right) v_{r}-\left(\begin{array}{c}
\left.\Delta v_{r} \partial_{r}+\frac{\Delta v_{\theta}}{r} \partial_{\theta}+\Delta v_{z} \partial_{z}+\frac{\Delta v_{r}}{r}+\frac{\partial_{\theta} \Delta v_{\theta}}{r}\right) v_{\theta} \\
-v_{z} \partial_{z} \Delta v_{\theta},
\end{array}\right.
$$

which implies a contribution to the growth rate of

$$
\left\langle\tilde{v}_{\theta}|\Delta L| A\right\rangle=\frac{\sin (2 \psi)}{8} \int_{-\infty}^{\infty} \tilde{v}_{\theta}\left(v_{r}\left(\partial_{r} \Delta v_{\theta}+\frac{\Delta v_{\theta}}{r}\right)+v_{\theta}\left(-\frac{1}{2} \partial_{r} \Delta v_{r}+\frac{\Delta v_{r}}{r}-\frac{\Delta v_{\theta}}{r}\right)\right.
$$




$$
\begin{gathered}
\left.+\Delta v_{z} \partial_{z} v_{\theta}\right) d z \\
=\quad \frac{\sin (2 \psi)}{8} \int_{-\infty}^{\infty} \tilde{v}_{\theta}\left(v_{r}\left(\frac{\Omega}{2} \partial_{r} \xi_{r}+2 \bar{\omega} \partial_{r} \xi_{\theta}-\frac{\Omega}{4} \frac{\xi_{r}}{r}+(2 \bar{\omega}-3 \Omega) \frac{\xi_{\theta}}{r}\right)\right. \\
+v_{\theta}\left(-\frac{\Omega}{2} \partial_{r} \xi_{\theta}+\bar{\omega} \partial_{r} \xi_{r}+\left(\frac{7}{4} \Omega-2 \bar{\omega}\right) \frac{\xi_{\theta}}{r}-2(\Omega+\bar{\omega}) \frac{\xi_{r}}{r}\right) \\
\left.-2 \bar{\omega} \xi_{z} \partial_{z} v_{\theta}\right) d z .
\end{gathered}
$$

The tidally perturbed vertical acceleration equation is

$$
\begin{array}{cc}
\partial_{t} v_{z}= & -S\left(\partial_{z} \ln \left(\frac{S}{\rho}\right)+\partial_{z}\right) \chi-z \Omega^{2} \delta \\
-v_{r} \partial_{r} \Delta v_{z}-\frac{1}{r} \partial_{\theta} \Delta v_{z}-v_{z} \partial_{z} \Delta v_{z}-(\Delta \vec{v} \cdot \vec{\nabla}) v_{z} \\
+\Delta \rho S\left(-\frac{z \Omega^{2}}{c_{s}^{2}}+\partial_{z}\right) \chi+\frac{\delta}{\rho} \partial_{z} \Delta P+2 \delta \frac{\Delta \rho}{\rho} z \Omega^{2}
\end{array}
$$

which implies a contribution to the growth rate of

$$
\begin{gathered}
\frac{\sin (2 \psi)}{8} \int_{-\infty}^{\infty} \tilde{v}_{z}\left(v_{z}\left(-\partial_{z} \Delta v_{z}+\frac{1}{2} \partial_{r} \Delta v_{r}-\frac{\Delta v_{\theta}}{r}\right)-\Delta v_{z} \partial_{z} v_{z}\right. \\
\left.-\frac{\Delta \rho}{\rho} S\left(-\frac{z \Omega^{2}}{c_{s}^{2}}+\partial_{z}\right) \chi-\delta\left(\frac{1}{\rho} \partial_{z} \Delta P+2 \frac{\Delta \rho}{\rho} z \Omega^{2}\right)\right) d z \\
=\frac{\sin (2 \psi)}{8} \int_{-\infty}^{\infty} \tilde{v}_{z}\left(v_{z}\left(\frac{\Omega}{2} \partial_{r} \xi_{\theta}-\bar{\omega} \partial_{r} \xi_{r}+\Omega \frac{\xi_{r}}{r}-\left(\frac{3}{4} \Omega+2 \bar{\omega}\right) \frac{\xi_{\theta}}{r}+2 \bar{\omega} \partial_{z} \xi_{z}\right)\right. \\
+2 \bar{\omega} \xi_{z} \partial_{z} v_{z}-\frac{\Delta \rho}{\rho} S\left(-\frac{z \Omega^{2}}{c_{s}^{2}}+\partial_{z}\right) \chi \\
\left.+z \Omega^{2}\left((\gamma-2) \frac{\Delta \rho}{\rho}+\gamma \frac{\Delta S}{S}-\partial_{z} \xi_{z}\left(2+z \partial_{z} \ln \rho+\frac{\gamma z^{2} \Omega^{2}}{c_{s}^{2}}\right)\right) \delta\right) d z .
\end{gathered}
$$

The tidally perturbed pressure evolution equation is

$$
\begin{aligned}
& \partial_{t} \chi=\quad-\frac{c_{s}^{2}}{S} \partial_{r} v_{r}+\frac{1}{S}\left(z \Omega^{2} v_{z}-c_{s}^{2} \partial_{z} v_{z}\right) \\
& +P^{-1 / \gamma}\left((-\vec{v} \cdot \vec{\nabla}) \Delta P-\gamma \Delta P \vec{\nabla} \cdot \vec{v}-\Delta \vec{v} \cdot \vec{\nabla}\left(P^{1 / \gamma} \chi\right)-\gamma \vec{\nabla} \cdot(\Delta \vec{v}) P^{1 / \gamma} \chi\right) \text {, }
\end{aligned}
$$

which implies a contribution to the growth rate of

$$
\begin{aligned}
\langle\tilde{\chi}|\Delta L| A\rangle= & \frac{\sin (2 \psi)}{8} \int_{-\infty}^{\infty} \tilde{\chi}\left(-\frac{c_{s}^{2}}{S} \frac{\Delta P}{P} k_{r} v_{r}+\frac{c_{s}^{2}}{S}\left(\frac{\partial_{z} \Delta P}{\gamma P}+\frac{\Delta P}{P} \partial_{z}\right) v_{z}\right. \\
& \left.\left(\left(\gamma-\frac{1}{2}\right)\left(\frac{\partial_{r}\left(r \Delta v_{r}\right)}{r}-\frac{2 \Delta v_{\theta}}{r}\right)+\Delta v_{z}\left(-\frac{z \Omega^{2}}{c_{s}^{2}}+\partial_{z}\right)+\gamma \partial_{z}\left(\Delta v_{z}\right)\right) \chi\right) d z \\
= & \frac{\sin (2 \psi)}{8} \int_{-\infty}^{\infty} \tilde{\chi}\left(-\frac{c_{s}^{2}}{S} k_{r} \frac{\Delta P}{P} v_{r}-\frac{z \Omega^{2}}{S}\left(\frac{\Delta P}{P}-\partial_{z} \xi_{z}\left(2+z \partial_{z} \ln \rho+\frac{z^{2} \gamma}{c_{s}^{2}}\right)\right) v_{z}\right. \\
+\frac{c_{s}^{2}}{S} \frac{\Delta P}{P} \frac{d v_{z}}{d z}+\chi\left(2 \bar{\omega}\left(\frac{z^{2} \Omega^{2}}{c_{s}^{2}}-\gamma\right) \partial_{z} \xi_{z}+\right. & \left.\left.\left(\gamma-\frac{1}{2}\right)\left(2 \Omega_{b} \frac{\xi_{r}}{r}-\xi_{\theta}\left(\frac{\Omega}{2}+4 \bar{\omega}\right)+\Omega \partial_{r} \xi_{\theta}-2 \bar{\omega} \partial_{r} \xi_{r}\right)\right)-2 \bar{\omega} \xi_{z} \frac{d \chi}{d z}\right) d z \\
& (\gamma
\end{aligned}
$$


Finally, the tidally perturbed density evolution equation is

$$
\begin{aligned}
& \partial_{t} \delta=\quad-\partial_{r} v_{r}-v_{z} \partial_{z} \ln (\rho)-\partial_{z} v_{z}-\frac{\Delta \rho}{\rho}\left(\partial_{r} v_{r}+\frac{v_{r}}{r}\right)-\partial_{r}\left(\frac{\Delta \rho}{\rho}\right) v_{r} \\
& -\frac{\Delta \rho}{\rho} \frac{1}{r} \partial_{\theta} v_{\theta}-\frac{v_{\theta}}{r} \partial_{r}\left(\frac{\Delta \rho}{\rho}\right)-\frac{\Delta \rho}{\rho} \partial_{z} v_{z}-\frac{v_{z}}{\rho} \partial_{z} \Delta \rho-\delta\left(\vec{\nabla} \cdot \Delta \vec{v}+\Delta v_{z} \partial_{z} \ln \rho\right) \text {. }
\end{aligned}
$$

which implies a contribution to the growth rate of

$$
\begin{gathered}
\left.\frac{\sin (2 \psi)}{8} \int_{-\infty}^{\infty} \tilde{\delta}|\Delta L| A\right\rangle=\frac{\Delta \rho}{\rho} k_{r} v_{r}+v_{z} \frac{\partial_{z} \Delta \rho}{\rho}+\frac{\Delta \rho}{\rho} \partial_{z} v_{z} \\
\left.+\left(\frac{1}{2 r} \partial_{r}\left(r \Delta v_{r}\right)-\frac{\Delta v_{\theta}}{r}+\Delta v_{z} \partial_{z} \ln \rho\right) \delta+\Delta v_{z} \partial_{z} \delta\right) d z \\
=\frac{\sin (2 \psi)}{8} \int_{-\infty}^{\infty} \tilde{\delta}\left(-\frac{\Delta \rho}{\rho} k_{r} v_{r}+v_{z}\left(-\partial_{z} \xi_{z}\left(\partial_{z} \ln \rho-z\left(\partial_{z} \ln \rho\right)^{2}+z \partial_{z}^{2} \ln \rho\right)+\frac{\Delta \rho}{\rho} \partial_{z} \ln \rho\right)\right. \\
+\frac{\Delta \rho}{\rho} \frac{d v_{z}}{d z}+\delta\left(\left(-\frac{1}{4} \Omega+2 \bar{\omega}\right) \frac{\xi_{\theta}}{r}+(\Omega-\bar{\omega}) \frac{\xi_{r}}{r}+\frac{\Omega}{2} \partial_{r} \xi_{\theta}-\bar{\omega} \partial_{r} \xi_{r}-2 \bar{\omega} \xi_{z} \partial_{z} \ln \rho\right) \\
\left.-2 \bar{\omega} \xi_{z} \frac{d \rho}{d z}\right) d z
\end{gathered}
$$

In order to calculate the growth rate for a given vertical structure, we need to solve for the vertical eigenfunctions using equations (10) through (15), and the adjoint eigenfunctions

using equations (19) through (24) or, more economically using equation (25), and substitute these results into equations (44), (46), (48), (50), and (52) using equations (37), (38) and (39). Finally, we need to add up the matrix components and divide by the state norm given by

$$
\langle\tilde{A} A\rangle=\frac{1}{4} \int_{-\infty}^{\infty}\left(\tilde{v}_{r} v_{r}+\tilde{v}_{\theta} v_{\theta}+\tilde{v}_{z} v_{z}+\tilde{\chi} \chi+\tilde{\delta} \delta\right) d z
$$

\section{Results}

We are now in a position to calculate the growth rates for the parametric tidal instability for a range of input parameters. Although our formalism allows us to take any vertical structure we will confine ourselves to presenting results only for adiabatic and isothermal vertical structures. These two cases should give us a sense of the range of possible results. We start with adiabatic disks. In this case the vertical structure is given by

$$
\begin{aligned}
& P=P_{0}\left(1-\left(\frac{z}{H}\right)^{2}\right)^{\frac{\gamma_{s}}{\gamma_{s}-1}}, \\
& \rho=\rho_{0}\left(1-\left(\frac{z}{H}\right)^{2}\right)^{\frac{1}{\gamma_{s}-1}},
\end{aligned}
$$


with

$$
\begin{gathered}
H=\left(\frac{2 P_{0}}{\rho_{0} \Omega^{2}}\right)^{1 / 2}, \\
c_{s}^{2}=\gamma \frac{P_{0}}{\rho_{0}}\left(1-\left(\frac{z}{H}\right)^{2}\right),
\end{gathered}
$$

and

$$
N^{2}=0
$$

The adiabatic index for the vertical structure is not necessarily the same index that appears in the perturbation equations. The latter describes the response of fluid elements on short time scales to changes in the ambient pressure, whereas the former is an attempt to model the vertical structure. In the preceding equations we have distinguished the two by using $\gamma_{s}$ when we mean the vertical structure parameter. This class of models has the peculiar property that the boundary occurs at $|z|=H$ rather than $|z|=\infty$. Consequently we have to modify our boundary conditions and take the solution which is least divergent near $|z|=H$ i.e. $\chi$ goes to a constant value near the vertical disk boundaries. An unfortunate consequence is that the fluid velocities are also constant, while the sound speed goes to zero. This is manifestly unphysical, but not too surprising. One expects the adiabatic approximation to break down far from the disk midplane. Fortunately the results of our calculation are weighted by the mode energy, and therefore by the local density, which drops to zero faster than the sound speed. It seems safe to assume that adding on an isothermal atmosphere will have little impact on our results as long as the column density in the atmosphere remains a small fraction of the total column density.

In figure 1 we show the growth rate for the parametric instability in units of $q \Omega_{b}$ for an adiabatic disk with $\gamma_{s}=\gamma=5 / 3$. as a function of $r / a$, where $a$ is the semi-major axis of the binary orbit. In this figure we plot the results for the three lowest order modes of internal waves. The primary mode has one zero in $\chi$ and none in $v_{z}$, i.e. the disk material bobs up and down as the wave passes by. In fact, in the limit where $\gamma_{s}=\gamma=1$, $v_{z}$ is not a function of $z$ at all. (However, in this case $v_{r}$ does have a vertical mode. The material above and below the midplane are moving in opposite directions radially during each cycle.) We see that the second and third harmonics are almost indistinguishable in this plot, and the primary mode shows large differences only for radii close to, or greater than, the vertical resonance radius. The fundamental mode shows a stronger response to the vertical resonance and a significantly larger growth rate for larger radii. At small radial distances our results converge to Goodman's asymptotic formula for the higher order modes. The primary mode is weaker by about $23 \%$. The vertical resonance peak is basically similar to the peak in his plot, but the zero in the growth rate occurs at a radius just outside the resonant radius, whereas in his calculation it was on the opposite side of the peak. The 
source of this discrepancy is not clear. It is not due to our disagreement concerning the sign of the $\Phi_{2}$ terms in equation (33).

In figure 2 we show the growth rate in units of $q \Omega_{b}$ for the fundamental mode and for $\gamma=\gamma_{s}=5 / 3,1.2$ and 1.0. This last case is equivalent to taking an isothermal vertical structure and assuming isothermal perturbations. The two most significant effects of changing the adiabatic index are that the vertical resonance is pushed outwards and the peak around it is broadened.

A more realistic, but still idealized, model is to assume an isothermal vertical structure for the disk. In this case we have $P \propto \rho, S \propto \rho^{1-1 / \gamma}$,

$$
\begin{gathered}
\rho=\rho_{0} \exp \left(\frac{-\rho z^{2} \Omega^{2}}{2 P}\right), \\
c_{s}^{2}=\gamma \frac{P}{\rho}
\end{gathered}
$$

and

$$
N^{2}=\frac{\rho z^{2} \Omega^{4}}{P}\left(1-\frac{1}{\gamma}\right)
$$

In figure 3 we show the growth rate in units of $q \Omega_{b}$ for the first three internal wave modes for an isothermal disk with $\gamma=5 / 3$. We see that the response to the vertical resonance is much larger in this case, by a factor of about $5 / 2$ for the fundamental mode. This carries over into a smaller response well beyond the radius of the vertical resonance. At much smaller radii the growth rates are similar to the adiabatic case, the only difference being that the primary mode is reduced from the higher order modes even more, by about $36 \%$.

In figure 4 we show the growth rate in units of $q \Omega_{b}$ for the fundamental mode in an isothermal disk with $\gamma=5 / 3,1.2$ and 1.0. As before, lowering $\gamma$ broadens the vertical resonance peak and moves it outward.

The modest differences between this calculation and Goodman (1993) for the adiabatic disk with $\gamma=5 / 3$ are explainable in terms of the additional effects included in this paper. On the other hand, our results are very different from Lubow et al. (1993). We have used a different calculational approach, and included the tidally induced vertical motions and pressure and density fluctuations. However, while these effects are quantitatively important, leaving them out does not produce a dramatic reduction in our growth rates, except near the vertical resonance. The most important difference seems to be our inclusion of radial derivatives in the tidal stresses. We can make a crude comparison between our two calculations by setting $\Delta \rho=\Delta P=\xi_{z}=0$, and taking $\partial_{r} \xi_{r}=\xi_{r} / r$ and $\partial_{r} \xi_{\theta}=\xi_{\theta} / r$. 
In figure 5 we show the growth rates for the fundamental mode in an isothermal disk with $\gamma=5 / 3$. The top curve shows the results of our complete calculation, also seen in figure 3. The next curve shows the results of removing tidally induced vertical motions, and density and pressure fluctuations. The bottom curve shows the results adding to this the assumption that the streamlines are locally self-similar, i.e. the radial derivatives of $\vec{\xi}$ are found by setting $\xi_{r}$ and $\xi_{\theta}$ proportional to $r$ in the interaction matrix. We note that at small $r$ the bottom curve is more than a factor of 5 below the top one. This is a dramatic reduction, although this result still lies about $30 \%$ above the the reduction obtained by Lubow et al.. The remaining discrepancy is apparently due to the difficulty in entering precisely equivalent assumptions into our program, due to our somewhat different methods for evaluating the interaction matrix. We conclude that the bulk of the difference between the results of Goodman (1993) and Lubow et al. (1993) stems from the latter's neglect of radial derivatives of the streamline perturbations, not from their inclusion of vertical structure.

\section{Conclusions}

We have calculated the linear growth rate of a tidally induced parametric instability in accretion disks. Our results take into account only leading order terms in the tidal distortion of an accretion disk, but include compressibility, vertical structure, vertical motions, and radial derivatives of the tidal distortions. Our basic conclusion is that the original results of Goodman (1993), derived under a simpler set of assumptions, are basically correct. For a disk with adiabatic vertical structure we see only small deviations except near the vertical resonance, where we find the zero in the growth rate lies just outside the radius of the vertical resonance, rather than just inside it. This difference is unlikely to have any significant dynamical effects. For a disk with isothermal vertical structure we find a general enhancement of the growth rate near the vertical resonance, by a factor of about 2.5. This implies that the net torque on an accretion disk may depend significantly on its vertical structure.

One lingering question regarding these results is the meaning of the infinite growth rate for the instability in the neighborhood of the vertical resonance. This singularity is a consequence of the divergence in $\xi_{z}$ at that radius. In reality there are two effects which

limit this divergence. First, strong vertical motions within a narrow annulus will lead to the emission of sound waves (Lubow 1981). First, equation (2) was derived neglecting radial 
gradients. If we define an effective radial wavenumber for the growth rate by

$$
K_{r} \equiv \partial_{r} \ln \sigma(r),
$$

then the tidal pumping of the vertical resonance will saturate due to the emission of sound waves from the resonant region when

$$
K_{r} V_{\text {group }}=\frac{c_{s}^{2} K_{r}^{2}}{2 \bar{\omega}} \approx \sigma(r) .
$$

Near the vertical resonance the growth rate is approximately

$$
\sigma(r) \approx A\left(\frac{r}{\Delta r}\right) q \frac{\Omega_{b}^{2}}{\Omega},
$$

where $\Delta r$ is the distance to the resonant radius and $A$ is a function of $\gamma$ and the vertical disk structure. We see that the half-width of the annulus within which damping by acoustic emission is important is

$$
\Delta r \approx \frac{\Omega}{2\left(\Omega-\Omega_{b}\right)} \frac{c_{s}^{2}}{q A(\gamma) \Omega_{b}^{2} r} \sim \frac{H^{2}}{q r A},
$$

where $H \sim c_{s} / \Omega$ is the disk thickness and we have ignored the distinction between $\Omega_{b}$ and $\Omega$ in the last (highly approximate) expression. Within this annulus the effective growth rate is

$$
\sigma_{l i m} \approx \frac{2 r^{2}\left(\Omega-\Omega_{b}\right)}{c_{s}^{2}}\left(A q \frac{\Omega_{b}^{2}}{\Omega}\right)^{2} \sim(q A)^{2} \Omega_{b}\left(\frac{r}{H}\right)^{2} .
$$

We see that as $(H / r) \rightarrow 0$ acoustic emission becomes ineffective at removing the effects of this singularity.

The second damping mechanism is due to nonlinear broadening of the vertical resonance. The resonance width will increase as the advective terms in the acceleration equation become important. In physical terms, the strong vertical oscillations of the disk will excite higher order harmonics, which will interfere with the resonant excitation of the fundamental mode. This is a purely one dimensional effect and does not depend on radial gradients in the tidal forcing. We can approximate its effects by inserting a term of the form $C_{0} 4 \bar{\omega}^{2}\left(\partial_{z} \xi_{z}\right)^{2}$ into the denominator of equation (33) where $C_{0}$ is a constant of order unity. This implies a limit on $\partial_{z} \xi_{z}$ of order

$$
\left(\partial_{z} \xi_{z}\right)_{l i m} \sim\left(q \frac{\Omega_{b}^{2}}{\Omega^{2}}\right)^{1 / 3} .
$$


Since the denominator of the leading factor in equation (33) goes as $-12 \Omega_{b} \bar{\omega}(\Delta r / r)$ this implies that this damping mechanism will be effective within an annular width of approximately

$$
\Delta r \approx r \frac{C_{0} \bar{\omega}}{12 \Omega_{b}}\left(\partial_{z} \xi_{z}\right)_{\text {lim }}^{2} \sim r \frac{C_{0} \bar{\omega}}{12 \Omega_{b}}\left(q \frac{\Omega_{b}^{2}}{\Omega^{2}}\right)^{2 / 3} .
$$

The instability growth rate within this annulus will be limited to

$$
\sigma_{l i m} \sim A\left(q \frac{\Omega_{b}^{2}}{\Omega^{2}}\right)^{5 / 3} \frac{12 \Omega_{b} \Omega}{C_{0}\left(\Omega-\Omega_{b}\right)} .
$$

Comparing equations (65) and (68) we note that acoustic emission will tend to be the most important damping process when the disk is not too thin, or when the tidal instability is driven only weakly. There is no general rule as to which will be more important in accretion disks in compact binary systems, and it is possible that for some systems acoustic emission may dominate during outbursts while nonlinear broadening is important during quiescence. In either case the ratio of the torque exerted within the annulus dominated by the vertical resonance to the torque exerted on the rest of the disk at a similar radius can be estimated by comparing the cube of the growth rate weighted by the annulus width (Goodman 1993). We have

$$
\frac{\sigma_{\lim }^{3} \Delta r}{\sigma(r)^{3} r} \sim\left(\frac{4 A}{15}\right)^{3}\left(\frac{r}{\Delta r}\right)^{2} .
$$

For $\gamma=5 / 3 A \approx 4.0$ for an isothermal disk and 1.7 for an adiabatic disk. This suggests that the vertical resonance can dominate the torque on a disk and that accretion disks will have a tendency to have an outer radius that lies within this annulus. This tendency should be particularly noticeable when the disk structure is isothermal and/or $\gamma$ is relatively small (so that $\Omega_{b} / \Omega$ is relatively large). However, apart from the vertical resonance $\sigma(r)$ rises sharply with $r$ and the torque per area, which goes as $H^{2} \sigma(r)^{3}$ rises even more sharply. This makes it possible for the dominant contribution to the torque on a disk to come from the outer edge of the disk provided that this lies a significantly greater radius than the vertical resonance.

In this paper we have assumed that the orbital motion of gas within the disk deviates from perfect circles only to the minimal extent required by tidal forces. For most systems this is a reasonable assumption, but there are particularly cases where it is likely to fail. For example, SU Uma stars exhibit superoutbursts which are probably due to the expansion of the outer edge of the disk through the $3: 1$ orbital resonance (Whitehurst 1988). Lubow (1991a, 1991b) has shown that at this radius the disk is vulnerable to an elliptical instability. Under these conditions the tidal parametric instability discussed here 
can exist as a secondary instability, implying a dramatic rise in the generation of internal waves during a superoutburst.

This work has been supported in part by NASA grant NAG5-2773. In addition, the author would like to thank the Harvard-Smithsonian Center for Astrophysics for their

hospitality while this paper was being written and Jeremy Goodman and Dongsu Ryu for several helpful conversations.

\section{REFERENCES}

Balbus, S.A., \& Hawley, J.F. 1991, ApJ, 376, 214

Bayly, B.J. 1986, Phys.Rev.Lett, 57, 2160

Brouwer, D., \& Clemence, G.M., 1961, Methods of Celestial Mechanics (New York: Academic Press)

Cannizzo, J.K. 1994, ApJ, 435, 389

Cannizzo, J.K. 1995, submitted to ApJ

Goodman, J. 1993, ApJ, 406, 596

Huang, M., \& Wheeler, J.C. 1989, ApJ, 343, 229

Landman, M.J., \& Saffman, P.G. 1987, Phys. Fluids, 30, 2339

Lubow, S.H. 1981, ApJ, 245, 274

Lubow, S.H. 1991a, ApJ, 381, 259

Lubow, S.H. 1991b, ApJ, 381, 268

Lubow, S.H., Pringle, J.E., \& Kerswell, R.R. 1993, ApJ, 419, 758

Meyer, F. \& Meyer-Hoffmeister, E. 1984, å, 132, 143

Mineshige, S., \& Wheeler, J.C. 1989, ApJ, 351, 47

Mineshige, S., \& Wood, J. 1989, MNRAS, 241, 59

Pierrehumbert, R.T. 1986, Phys.Rev.Lett, 57, 2157 
Ryu, D., \& Goodman, J., 1994, ApJ, 422, 269

Ryu, D., Goodman, J., \& Vishniac, E.T. 1995, submitted to ApJ

Smak, J.I. 1984a, Acta Astronomica, 34, 161

Smak, J.I. 1984b, PASP, 96, 5 34, 161

Vishniac, E.T., \& Diamond, P.H. 1989, ApJ, 347, 435

Vishniac, E.T., Jin, L., \& Diamond, P.H. 1990, ApJ, 365, 552

Vishniac, E.T., \& Diamond, P.H. 1992, ApJ, 398, 561

Vishniac, E.T., \& Diamond, P.H. 1994, in Accretion Disks in Compact Stellar Systems, ed. J.C. Wheeler (Singapore: World Scientific Press), 41

Whitehurst, R. 1988, MNRAS, 232, 35 


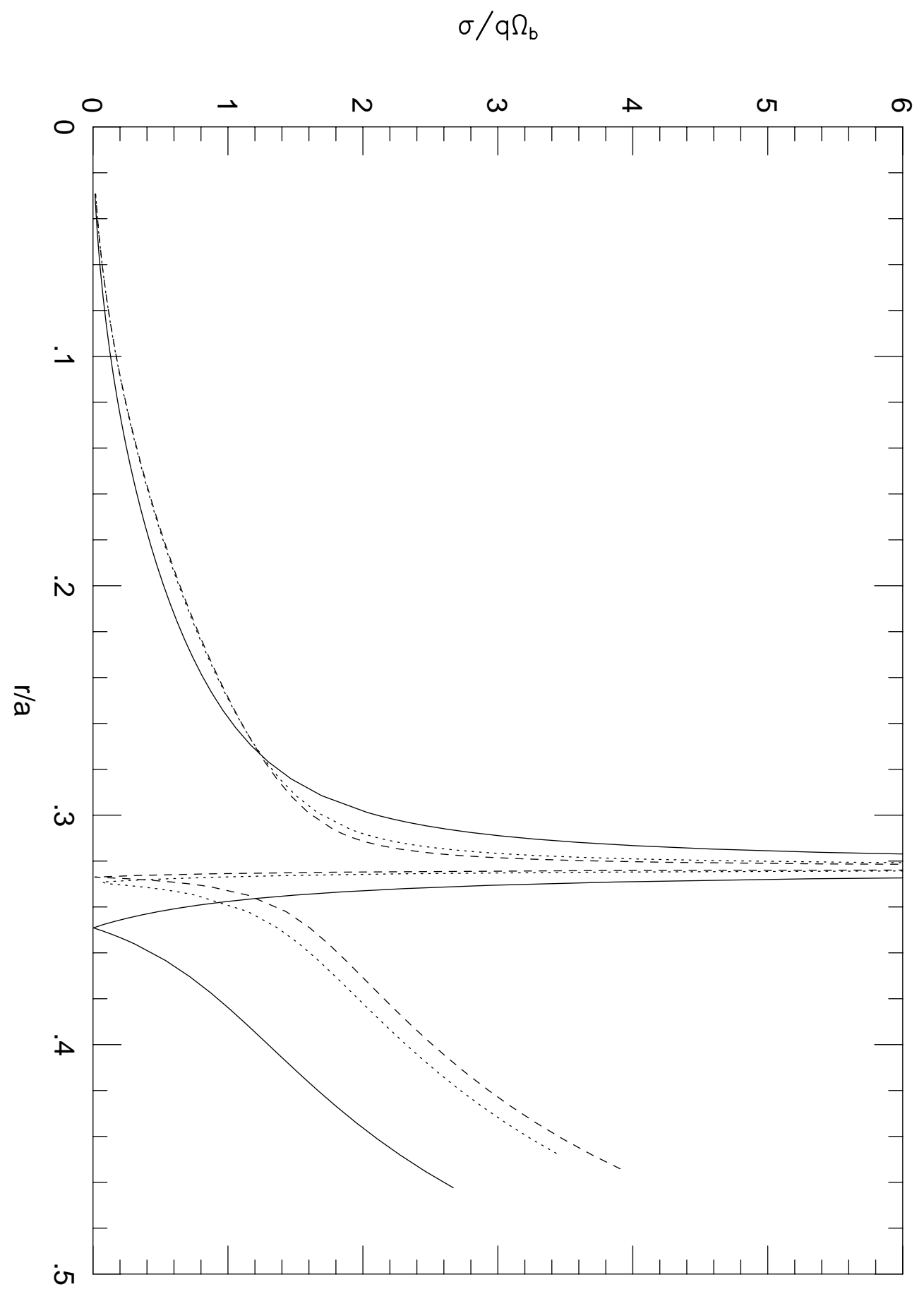

Fig. 1.- Growth rates for the parametric instability in units of $q \Omega_{b}$ as a function of $r / a$ for an adiabatic accretion disk with $\gamma=5 / 3$. The solid line is for the minimal vertical wavenumber. The dotted and dashed lines are for the second and third wavenumbers. 


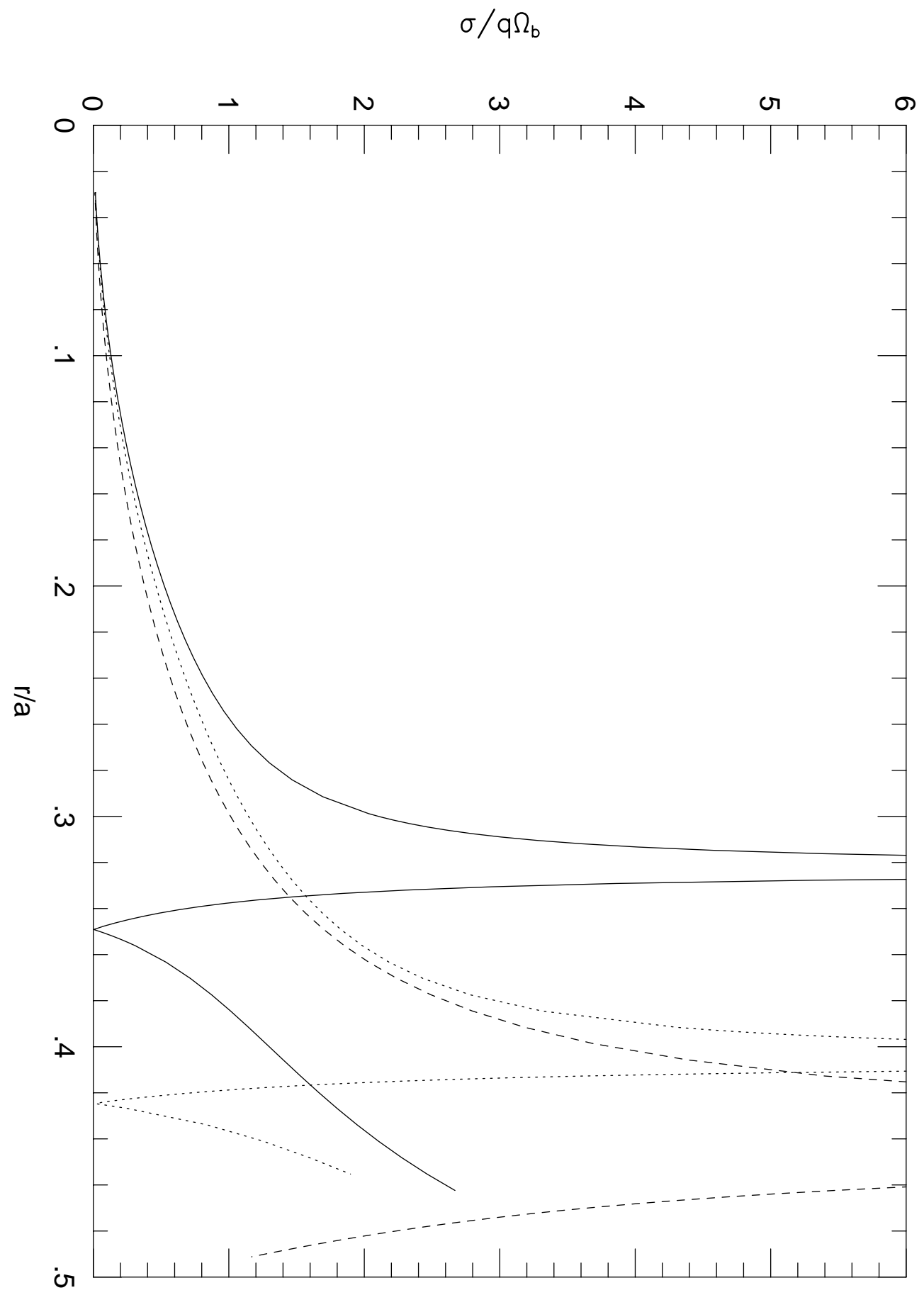

Fig. 2.- Growth rates for the minimal vertical wavenumber mode in an adiabatic disk for $\gamma=5 / 3$ (solid line), $\gamma=1.2$ (dotted line), and $\gamma=1.0$ (dashed line). 


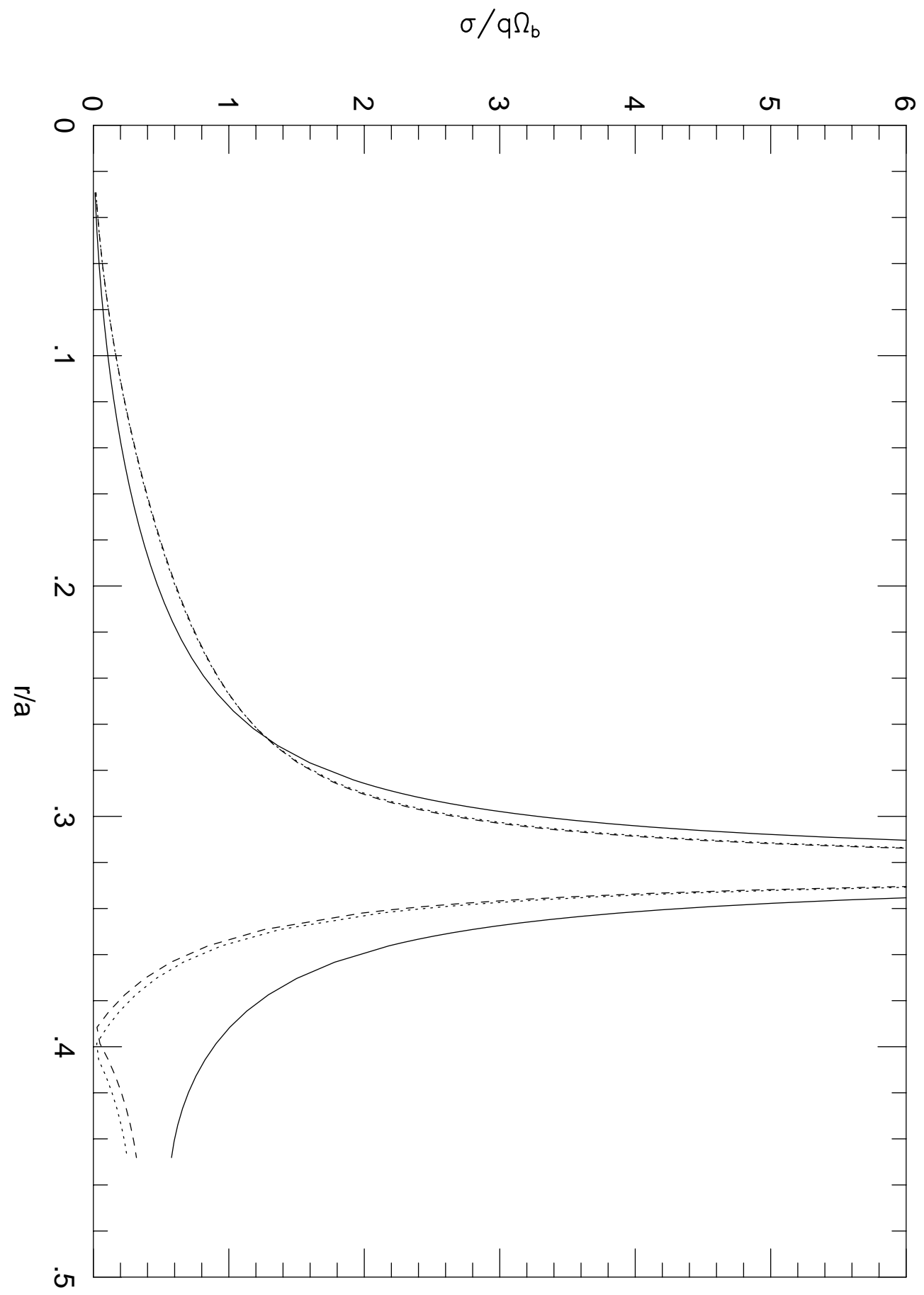

Fig. 3.- Growth rates for an accretion disk with an isothermal vertical structure and $\gamma=5 / 3$. The solid line is for the minimal vertical wavenumber. The dotted and dashed lines are for the second and third wavenumbers. 


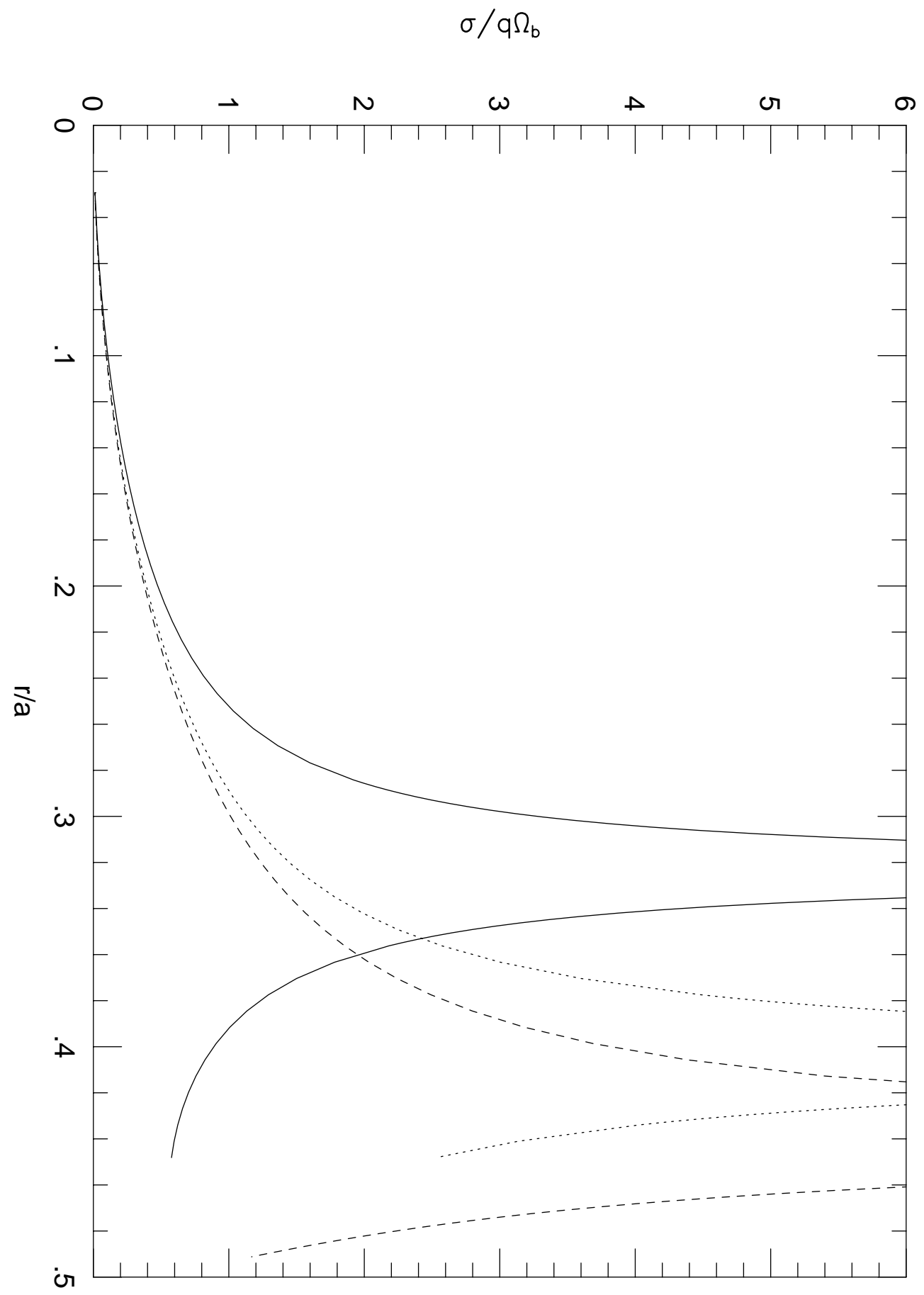

Fig. 4. - Growth rates for the minimal vertical wavenumber mode in an isothermal disk for $\gamma=5 / 3$ (solid line), $\gamma=1.2$ (dotted line), and $\gamma=1.0$ (dashed line). 


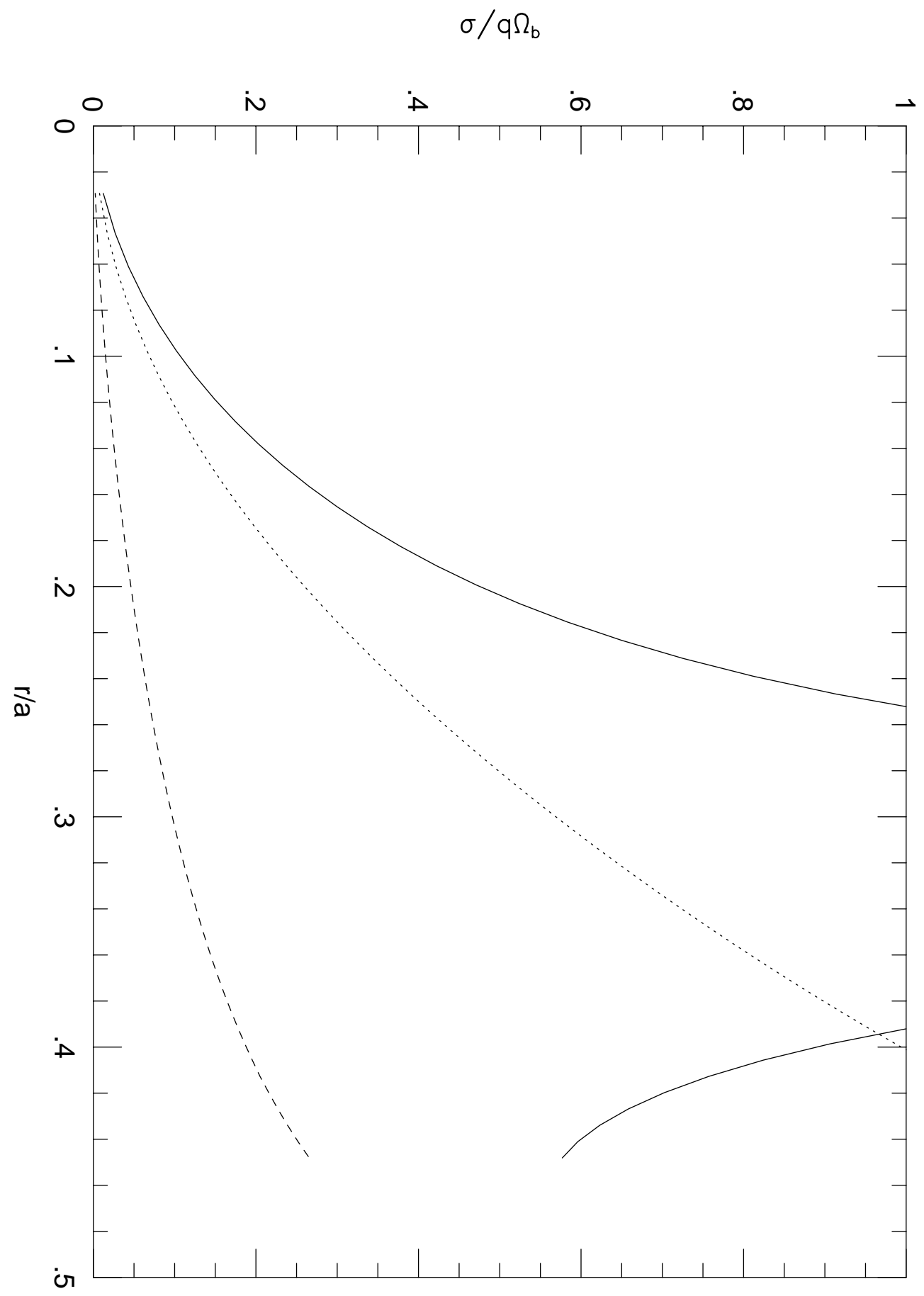

Fig. 5.- Growth rates for the minimal vertical wavenumber mode in an isothermal disk with $\gamma=5 / 3$. The solid line is full result. The dotted line shows the effect of suppressing vertical motion and tidal perturbations to density and pressure. The dashed line results from also assuming $\vec{\xi} \propto r$. 